\title{
Inverse Localization of Intraventricular Pacing Sites by Equivalent Dipole Source
}

\author{
Jana Svehlikova, Milan Tysler \\ Institute of Measurement Science, Slovak Academy of Sciences, Bratislava, Slovakia
}

\begin{abstract}
Localization of seven intraventricular pacing sites in the left ventricle was performed using a single dipole as equivalent heart generator.

The used data were provided by Karlsruhe Institute of Technology in EDGAR database. Body surface potential maps were measured in 63 precordial leads during intraventricular pacing with recorded position of the stimulating catheter. The inverse solution was computed for instantaneous and integral maps from the initial $30 \mathrm{~ms}$ time interval of ventricular activation. The role of the relative residual error between the input map and the map computed from the resulting dipole was studied with respect to the accuracy of the inverse solution.

The localization error of the obtained results for the initial $30 \mathrm{~ms}$ was dependent on the position of the pacing site and varied from $20-30 \mathrm{~mm}$ for anterior and lateral sites to $45-55 \mathrm{~mm}$ for inferior sites. The results were improved considerably when the considered time interval was prolonged and the inverse solution was computed for the time instant when the residual error reached its minimum.

The proper time instant for computation of the input data for localization of pacing sites should be chosen individually for each ECG signal.
\end{abstract}

\section{Introduction}

The presented study continues with processing the data provided in EDGAR database [1] which were prepared for applying the methods for localization of the starting points of the undesired ventricular activity. Noninvasive assessment of the source of a premature ventricular contraction (PVC) is the aim of various research groups e.g. [2], [3], [4]. It is expected that such knowledge can help to shorten the invasive ablation procedure when such source is eliminated. The problem leads to finding the proper inverse solution in electrocardiography, which represents an ill posed problem [5]. Besides the regularization techniques also the influence of the other factors such as the respiratory movement or the complexity of the torso model on the inverse problem solution have been studied [6], [7].
When using models of equivalent electrical heart generator in the form of transmembrane voltages, epicardial potentials or equivalent double layer [8], [9] the solution is computed for the whole myocardial volume or surface. Inverse solution using a single dipole [7] represents a different approach when the region activated by PVC at the beginning of the activation is represented by one dipole. In [10] this approach was applied on the data from the EDGAR database for localization of spontaneous PVC for one patient. In the presented study, the method was used for localization of controlled intraventricular pacing stimulations realized on the same subject.

\section{Materials and Methods}

The ECG signals measured by multiple leads measuring system were provided by Karlsruhe Institute of Technology and University Medical Centre Mannheim via EDGAR database [1]. Body surface potential maps (BSPMs) were recorded in 63 precordial leads in one patient from intraventricular pacing (IVP) during the ablation procedure. Seven positions of the IVP catheter (IVP1-IVP7) were given using the CARTO intracardiac mapping system (Figure 1 left) with the corresponding measured BSPMs. The geometry of the patient's torso as well as of the ventricular myocardium were obtained from MR scans and defined as closed triangular meshed surfaces. The scans were taken in the end-diastolic phase of cardiac cycle. The positions of the measuring electrodes with respect to the torso geometry were also provided (Figure 1 right).

The ECG recordings were performed at a sampling rate of $1 \mathrm{kHz}$. Fourteen and ten repetitive measurements were obtained from pacing sites IVP1 and IVP2 respectively. For all other pacing sites only one ECG recording was obtained.

BSPMs representing only the first $30 \mathrm{~ms}$ of ventricular activation were studied and evaluated. It is assumed that during that time interval the activated area is small enough to be represented by a single dipole, as used in the proposed inverse method. Two types of BSPMs were computed - instantaneous BSPMs computed for each time sample of the measurement within the studied interval, and integral BSPMs computed for all time 
intervals starting from the beginning of the activation and lasting from $1 \mathrm{~ms}$ up to the end of the studied interval (1$2 \mathrm{~ms}, 1-3 \mathrm{~ms}, \ldots 1-30 \mathrm{~ms})$.
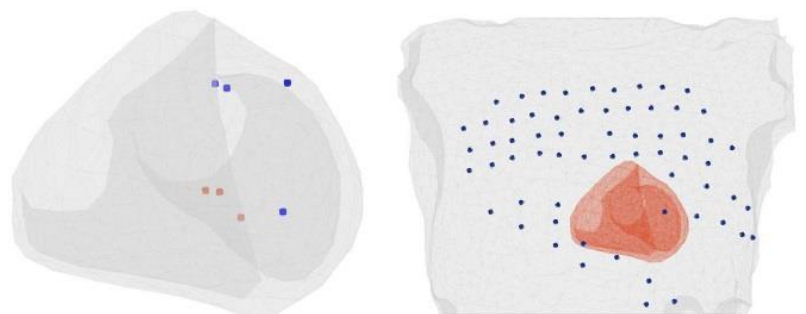

Figure 1. Left: The model of the surface of ventricular myocardium with the seven IVP sites in the left ventricle. The blue and red colors correspond with the accuracy of the IVP sites localization (see the Results). Right: Corresponding torso model with the positions of 63 measuring electrodes.

The inverse solution to one dipole is described in [10]. Briefly, a position of the dipole representing the activated area is searched in the group of predefined points within the model of the ventricular myocardium. In the presented work three sets of predefined positions for resulting dipoles were defined. The first set (endoepi) was defined on vertices of the triangulated surface of myocardium and consisted of 502 points. The second set (heartVol) was defined as 2223 points inside the myocardial surface. The third set (grid3) was constructed by filling the myocardial surface with points in a regular grid with mutual distance $3 \mathrm{~mm}$ what resulted in 10357 positions. For each input BSPM, the dipole best representing the input map was computed for all sets of predefined positions. Then the representative position for the origin of the measured PVC was chosen for the dipole creating the most similar BSPM on the torso to the input map. This similarity was evaluated by the relative residual error (RRE) parameter computed as:

$$
R R E=\frac{\| \text { measBSPM-compBSPM\| }}{\| \text { measBSPM\|}}
$$

where measBSPM is the measured input map and compBSPM is the map computed from the dipole estimated by the inverse solution. Vertical bars indicate the Euclidean norm of the vector.

The localization error (LE) was computed as the Euclidean distance between the defined position of intraventricular pacing and the inverse solution. The influence of the IVP placement, type of the input BSPM and the density of predefined positions for the inverse solution was studied. The relationship between the RRE parameter and the LE was also evaluated.

\section{Results}

The inverse solutions were computed for instantaneous and integral BSPMs for three sets of predefined positions for possible resulting dipoles within the ventricular volume. For each set, the model error as the distance between the defined position of IVP point and the closest member of the set was computed.

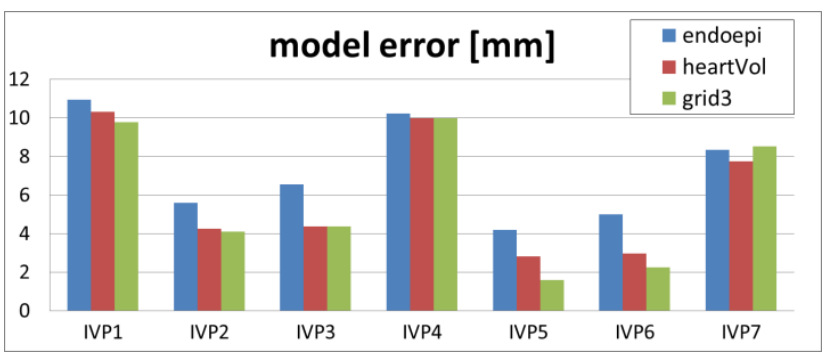

Figure 2. The model error of the stimulated points IVP1 - IVP7 with respect to the three sets of predefined positions for possible inverse localization of the dipole.

From all obtained inverse results the results were considered as relevant if the RRE value was less than 0.20 . The example of the value of the RRE parameter for the first $100 \mathrm{~ms}$ of the ventricular activation is shown in Figure 3.

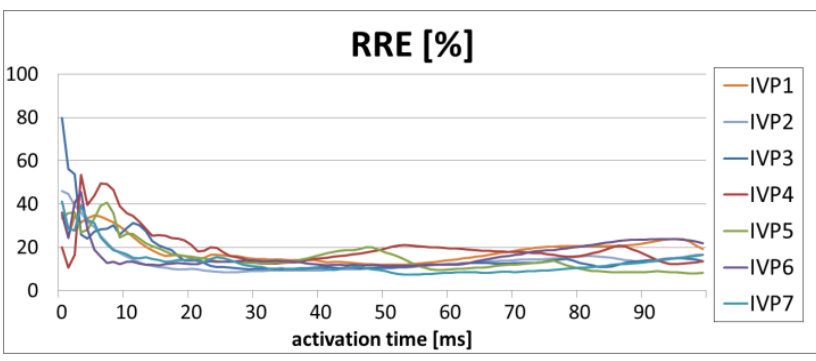

Figure 3. Value of the RRE parameter of the inverse solutions computed for instantaneous BSPMs up to 100 $\mathrm{ms}$ from the activation onset for predefined positions on the endo-epicardial surface.

The LE was first evaluated for the first $30 \mathrm{~ms}$ of the activation. The relevant results existed for all IVP pacing sites, all types of input BSPMs and all sets of predefined positions for resulting dipoles. The LE of all relevant results depending on the IVP pacing site placement and the type of the input BSPM is depicted in Figure 4.

From the obtained values of the LE there were two groups of results with respect to the true position of the IVP: First group: IVP2, IVP3, IVP4 and IVP5 with the mean LE about 20-30 mm; second group: IVP1, IVP6, and IVP7 with the mean LE about $45-55 \mathrm{~mm}$. The positions of the true IVP pacing sites from the first group were located anterior or inferolateral of the left ventricle and are illustrated by blue color on Figure 1 left. The 
second group of the true IVP positions was located inferiorly in the left ventricle near the base (red points on Figure 1 left).

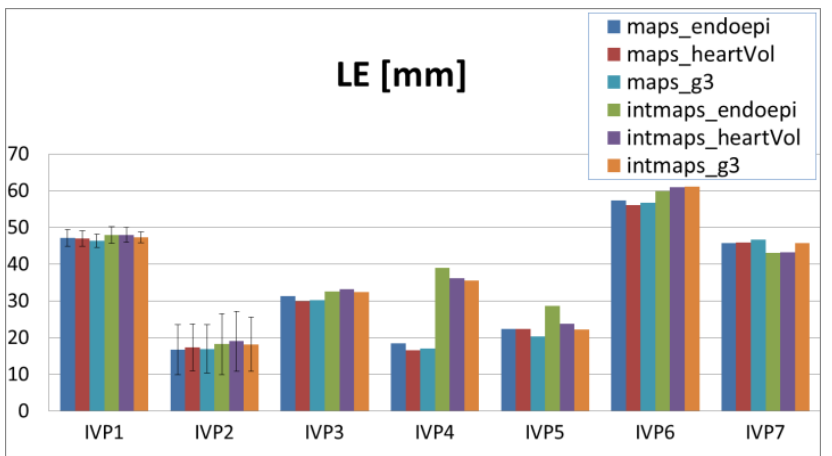

Figure 4. LE of all relevant results within the initial $30 \mathrm{~ms}$ time interval of activation. The error bars indicate the standard deviation of results for two cases when repetitive measurements were performed.

Next, to observe the relationship between the RRE parameter and the LE the inverse solution for instantaneous input BSPMs was computed for up to $100 \mathrm{~ms}$ from the beginning of activation. The development of the LE for predefined positions in grid3 is illustrated in Figure 5. The LE for the inferior IVP sites dropped down later than $50 \mathrm{~ms}$ after the activation onset.

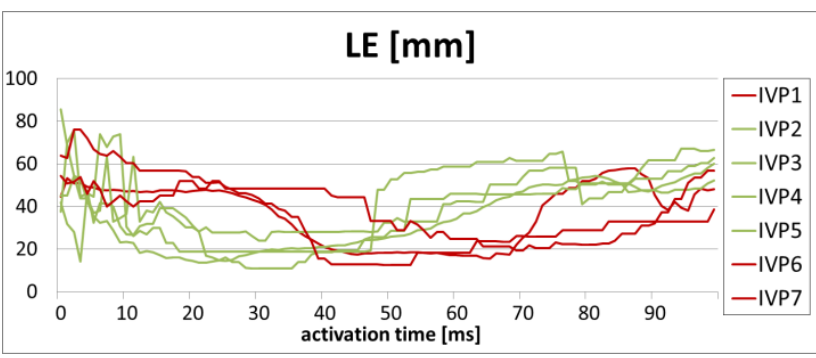

Figure 5. LE of the inverse solution from the input BSPMs computed for the first $100 \mathrm{~ms}$ from the activation onset. Green lines indicate the IVP positions with the best LE within the first $30 \mathrm{~ms}$, red lines show inferior IVP positions with the best LE for later activation time

When observing the RRE parameter together with the LE, the best values of the LE were obtained always near the minimal value of the RRE parameter. Therefore the LE for the input BSPM computed in the time instant when the minimal RRE value was obtained was also evaluated as shown in Figure 6. Because there were no differences in the results for integral and instantaneous input BSPMs for the initial $30 \mathrm{~ms}$ time interval, for the longer time interval only results for instantaneous BSPMs were evaluated.

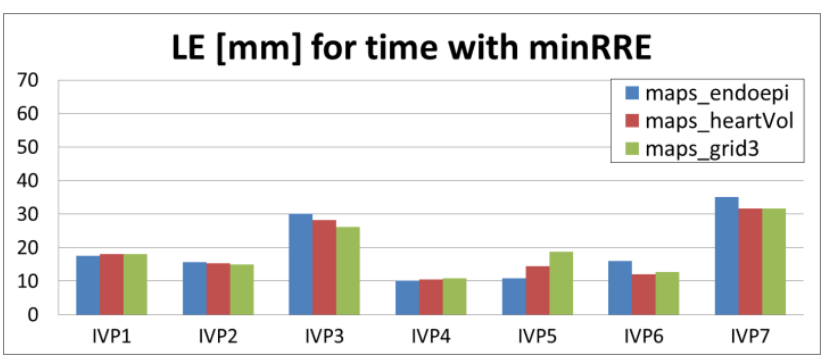

Figure 6. Localization error of the inverse solution computed from the BSPM in the time with the minimal value of the RRE parameter.

\section{Discussion}

Surprisingly as it can be seen in Figure 2 for some IVP positions the model error did not decrease with the higher density of the predefined positions as would be expected. After visual inspection of the IVP positions, it was revealed that with respect to the given triangulated surface of the ventricles the IVP origins were not defined inside the surface but they were placed inside of the ventricular cavity.

Considering the type of the input BSPM there were no differences in inverse results if the instantaneous or integral maps were used except for one case when the IVP4 pacing site was stimulated. The integration from the beginning of the studied interval should decrease the random noise from the signal however on the other hand in such a map the very initial maps with small values are non-dipolar (e.g. see Figure 3, IVP3) and produce additional "space" noise that is summed in the integral. Therefore the integration did not contribute to the improvement of the final result.

The higher density of the predefined positions for the inversely computed dipole representing the IVP pacing site did not lead to lower LE. The only parameter influencing the quality of the inverse IVP localization was the position of the pacing site. All solutions with unacceptable large LE $45-55 \mathrm{~mm}$ were obtained for the IVP pacing sites from the inferior wall of the left ventricle. The computed results were projected to the anterior wall of the left ventricle. One of the reasons can be the only precordial and very irregular placement of the measuring electrodes.

The length of the studied time interval was set generally as initial $30 \mathrm{~ms}$ to represent the initial activated area by a single dipole. From the obtained results it appeared that for some IVP locations better results were obtained using longer time interval. The minimal LE was always obtained near the time instant with minimal RRE value. Thus the minimal RRE value could be an indicator of the most appropriate time instant for computation of the input BSPM for the inverse solution. Although the activated area in later time instant is not very small, probably the position of its representative dipole is still 
near the origin of activation. When the LE was evaluated according the criterion of the minimal RRE value the localization of IVP origins improved considerably as it is shown in Figure 6. The individual approach to ECG signal processing could improve the inverse localization. Similar results were shown also in [11] where the time instants for inverse computation were set individually according to the length of the QRS interval and R-peak value and the best inverse results were also obtained for later time instants.

The results obtained in this study are also in agreement with the results in [10] when for the same patient and the same configuration of measuring electrodes the spontaneous premature ventricular contraction started on the anterior side near the base and was localized by the same method with the LE up to $25 \mathrm{~mm}$.

\section{Conclusion}

A single dipole model as the equivalent heart generator was used for localization of the seven positions of intraventricular pacing sites on the left ventricle.

First the inverse solution was computed from the initial $30 \mathrm{~ms}$ time interval of activation. It was shown that the use of integral BSPMs instead of instantaneous BSPMs did not improve the results. The localization error was less than $30 \mathrm{~mm}$ for the four pacing sites placed on the anterior or lateral side of the left ventricle. For the three pacing sites with the inferior position the results from the studied time interval were found on the anterior site of the left ventricle. The results up to $30 \mathrm{~ms}$ were obtained for all positions of the pacing sites if they were computed for the input map from the time instant when the minimal RRE value was computed.

The minimal value of RRE parameter can serve as the indicator for individual choice of the proper time for computing the input BSPM for evaluation of the results of inverse solution.

\section{Acknowledgements}

This work was supported by the research grant 2/0071/16 from the VEGA Grant Agency in Slovakia and by the grant APVV-14-0875 from the Slovak Research and Development Agency.

\section{References}

[1] Aras K, Good W, Tate J, Burton B, Brooks D, Coll-Font J, et al. Experimental Data and Geometric Analysis Repository - EDGAR. J Electrocardiol 2015;48:975-81.

[2] Rudy Y. Noninvasive electrocardiographic imaging of arrhythmogenic substrates in humans. Circ Res 2013;112:863-74.

[3] Cluitmans MJM, Peeters RLM, Westra RL, Volders PG a. Noninvasive reconstruction of cardiac electrical activity: update on current methods, applications and challenges. Netherlands Hear J 2015;23:301-11.

[4] Bear L, Cuculich PS, Bernus O, Efimov I, Dubois R. Introduction to noninvasive cardiac mapping. Card Electrophysiol Clin 2015;7.

[5] Tuboly G, Kozmann G, Maros I. Computational Aspects of Electrocardiological Inverse Solutions. IFACPapersOnLine 2015;48:48-51.

[6] Coll-Font J, Roig-Solvas B, Van Dam P, MacLeod RS, Brooks DH. Can we track respiratory movement of the heart from the ECG itself - and improve inverse solution too? J Electrocardiol 2016;49:927.

[7] Punshchykova O, Švehlíková J, Tyšler M, Grünes R, Sedova K, Osmančík P, et al. Influence of Torso Model Complexity on the Noninvasive Localization of Ectopic Ventricular Activity. Meas Sci Rev 2016:96-102.

[8] Potyagaylo D, Segel M, Schulze WHW, Dössel O. Noninvasive localization of ectopic foci: A new optimization approach for simultaneous reconstruction of transmembrane voltages and epicardial potentials. Lect Notes Comput Sci (Including Subser Lect Notes Artif Intell Lect Notes Bioinformatics) 2013;7945 LNCS:166-73.

[9] Van Dam PM, Tung R, Shivkumar K, Laks M. Quantitative localization of premature ventricular contractions using myocardial activation ECGI from the standard 12-lead electrocardiogram. J Electrocardiol 2013;46:574-9.

[10] Švehlíková J, Tyšler M. The Noninvasive Localization of Premature Ventricular Activity Using Different Equivalent Point Sources. Comput Cardiol Conf 2016;43.

[11] Schulze WHW. ECG Imaging of Ventricular Activity in Clinical Applications. PhD Thesis. Karlsruhe: KIT Scientific Publishing, Karlsruhe; 2015; p.105.

Address for correspondence:

Jana Svehlikova

Institute of Measurement Science, Slovak Academy of Sciences Dubravska cesta 9, 84104 Bratislava, Slovakia jana.svehlikova@savba.sk 